\title{
AIDS and COVID-19 are two diseases separated by a common lymphocytopenia
}

\section{Salvatore Sciacchitano ( $\nabla$ salvatore.sciacchitano@uniroma1.it )}

Sapienza University of Rome https://orcid.org/0000-0003-1492-5365

\section{Simonetta Giovagnoli}

Sapienza University of Rome

\section{Rachele Amodeo}

Sapienza University of Rome

lolanda Santino

Sapienza University of Rome

\section{Maurizio Simmaco}

Sapienza University of Rome

Paolo Anibaldi

Sant'Andrea Hospital Rome

\section{Deborah French}

Sapienza University of Rome

Rita Mancini

Sapienza University of Rome

Claudia De Vitis

Sapienza University of Rome

\section{Michela D'Ascanio}

Sapienza University of Rome

\section{Alberto Ricci}

Sapienza University of Rome

\section{Alfredo Pennica}

Sapienza University of Rome

\section{Antonio Aceti}

Sapienza University of Rome

\section{Research Article}

Keywords: SARS-CoV-2, HIV, Coronavirus disease 2019 (COVID-19), acquired immunodeficiency syndrome (AIDS), flow cytometric analysis

Posted Date: July 16th, 2020 
DOI: https://doi.org/10.21203/rs.3.rs-43462/v1

License: (a) (i) This work is licensed under a Creative Commons Attribution 4.0 International License. Read Full License 


\section{Abstract}

HIV and SARS-CoV-2 are responsible for two of the most dangerous and life-threatening infectious diseases of our times. To better analyze the difference in the immunological response elicited by the two infections, we compare the alterations in the lymphocyte subpopulations, measured by flow cytometry analysis (FCA) in both AIDS and COVID-19 patients, referred to our University Hospital. A total of 184 HIV infected patients were retrospectively examined and the results of FCA collected and compared to those obtained in 110 SARS-CoV-2 infected patients, examined during the actual outbreak. We observe a comparable reduction in B cells in both diseases and a more severe reduction in the total amount of $T$ cells in COVID-19 as compared to AIDS patients. The analysis of the T cells subpopulations indicates that there is a comparable reduction in the CD4+ cells count. Conversely, a remarkable difference between them is observed in the CD8+ counts. In AIDS patients the CD8+ cells are slightly higher than normal, while in COVID-19 patients the CD8+ cell count is markedly reduced. As a result, the CD4+/CD8+ ratios, is very low in AIDS and higher than normal in COVID-19 patients. The NK cells are reduced in both diseases, but SARS-CoV-2 infection causes a more severe reduction compared to HIV infection.

In conclusion, both HIV and SARS-CoV-2 viruses induce major changes in the lymphocytes count, with remarkable similarities and differences between them. The total absolute numbers of T cells and, in particular of the CD8+ subpopulation, are lower in COVID-19 patients compared to AIDS ones, while the $\mathrm{CD} 4+$ are reduced in both at similar levels. These results indicate that the host immune system reacts differently to the two infection, but they are responsible of a comparable dropping effect on the serum levels of CD4+ $T$ cell population. The meaning of the similarities and of the differences in terms of $T$ cells

activation and serum depletion are discussed. The knowledge on how the immune system reacts to these two infections will be useful to better define their mechanism of action and to design specific preventive and therapeutic approaches.

\section{Introduction}

The actual outbreak of SARS-CoV-2 virus has caused a pandemic disease, the coronavirus disease2019 (COVID-19), responsible for one the worst global health crisis. Not many years ago, we faced another serious public health challenge due to an infectious agent, namely the HIV, responsible for another global pandemic, the Acquired Immune Deficiency Syndrome (AIDS). Although HIV/AIDS and SARS-CoV-2/COVID-19 differ in their means of infection, disease courses and treatments, they both represent a health threat to the general population and both viruses are able to induce major changes in the lymphocytes count, with some similarities and striking differences between them, indicating that immune system reacts differently to the two infections.

Infection of humans by the human immunodeficiency virus (HIV) is known to be responsible for a progressive, multifactorial impairment of the immune system, eventually leading to the acquired 
immunodeficiency syndrome (AIDS). The defining features of the acquired immunodeficiency are the persistent and profound selective decrease in the function as well as number of T lymphocytes of the helper/inducer subset and a possible activation of the suppressor/cytotoxic subset, as described in 1982 in four patients [1].

Infection of humans by the SARS-CoV-2 virus is responsible for COVID-19, an acute respiratory failure, eventually leading to septic shock, and/or multiple organ dysfunction or failure [2]. The typical clinical presentation of COVID-19 consists in interstitial pneumonia, with fever, cough or chest tightness, anosmia, myalgia, fatigue and dyspnea. The common feature consisted in lymphopenia, which can be severe and progressive, especially in the more severely affected patients and in those with fatal illnesses [3]. In patients with COVID-19, both helper T cells (CD4+) and suppressor T cells (CD8+) have been reported to be below normal levels, and the decline of helper $\mathrm{T}$ cells was more pronounced in the most severe cases. There is also evidence of an exuberant inflammatory response, similar to cytokine release syndrome, which was defined as "cytokine storm syndrome" [4].

The aim of this study is to compare the results of flow cytometric analysis in patients infected by HIV and by SARS-CoV-2.

In addition to the many differences in structures, transmission modalities, mechanism of infections and clinical presentations, the HIV and SARS-CoV-2 show also different ability to elicit immune response, but they present some remarkable similarities. The analysis of the different effects of these two viruses on the host immune dysregulation will help us in understanding what pathogenic mechanisms lead to the debacle of the immune system observed in these two diseases.

\section{Materials And Methods}

\section{Study samples}

Both AIDS and COVID-19 patients referred to our University Hospital in Rome, Italy. They were examined at presentation of their disease.

\section{COVID-19 patients}

Blood samples were obtained from patients referred for COVID-19 during the actual SARS-CoV-2 outbreak. All patient had a positive test for SARS-CoV-2 virus, performed using nasopharyngeal and oropharyngeal swabs, followed by real-time reverse-transcription polymerase chain reaction (rRT-PCR), according to the WHO recommendation [5]. In particular, two rRT-PCR detection kits have been used (Allplex ${ }^{\mathrm{TM}}$ 2019-nCoV Assay, Seegene, Seul, Republic of Korea and RNA Detection kit, DAAN Gene Co. LTD, Guangzhou, Guandong, China), both CE-approved. The test has been considered positive in the presence of at least two of the three genes considered (E, RdRP and N genes) for the kit by Seegene and of the two genes considered (ORF1ab and $\mathrm{N}$ genes) for the kit by DAAN. 


\section{AIDS patients}

The results of cytofluorimetric assays from a total of patients affected by HIV and followed by our Dept. of Infectious disease were retrieved and analyzed. Diagnosis of HIV infection was given in agreement with the WHO guidelines [6]. Blood was drawn from all of them at the time of diagnosis for haemocrocytometric and flow cytometric analyses and data have been collected. They underwent highly sensitive HIV viral load assay to count the number of HIV particles in a milliliter $(\mathrm{mL})$ of blood (VERSANT ${ }^{\circledR}$ HIV-1 RNA 1.5 Assay, kPCR, Siemens Healthcare GmbH Erlangen, Germany). The test was considered positive for a value higher than 50 copies in one milliliter of blood.

\section{Flow Cytometric Analysis}

FCA was performed in the automated AQUIOS CL $®$ "load \& go" flow cytometer (Beckman Coulter, Life Sciences Division, Indianapolis, USA). The same instrument and the same reagents were used for both groups of patients to ensure the possibility to perform accurate comparative analysis.

\section{Statistical Analysis}

Descriptive statistical analysis was performed on raw data where applicable. The results are expressed as means \pm SD. A two-tailed $P$ value of 0.05 or less was used as a criterion to indicate statistical significance. NS = not significant. Data have been statistically analyzed using the GraphPad Prism software (version 8.4.1) (GraphPad Software, San Diego, CA).

\section{Ethical Approval}

A written informed consent was obtained by the participants to the study. The study was approved by our Institutional Ethical Committee (University La Sapienza of Rome, Italy) (Prot.\# 52SA_2020, RIF. CE 5773_2020), on the basis that it complied with the declaration of Helsinki and that the protocol followed existing good clinical practice guidelines.

\section{Results}

\section{Patient epidemiological data}

All patients have been seen at our University Hospital. Epidemiological data of both group of patients are reported in Table 1 (Table 1).

Table 1. Study population 


\begin{tabular}{|lll|}
\hline Characteristics & COVID-19 $(\mathrm{n} .=110)$ & AIDS (n. = 184) \\
\hline Age, years & $69(35-94)$ & $40(21-69)$ \\
\hline Sex & & \\
\hline Men & $67(61 \%)$ & $156(85 \%)$ \\
\hline Women & $43(39 \%)$ & $28(15 \%)$ \\
\hline F/M ratio & 0.6 & 0.2 \\
\hline Year of Diagnosis & 2020 & $1996-2019$ \\
\hline SARS-CoV-2 & Positive in all & \\
\hline HIV viral load test & & Positive in all \\
\hline
\end{tabular}

\begin{tabular}{|ll|}
\hline $\begin{array}{l}\text { number of copies/ml } \\
\text { (mean value } \pm \text { SD) }\end{array}$ & $(377,696 \pm 984,115)$ \\
\hline$<100,00$ copies/ml, n. $(\%)$ & $105(57.1 \%)$ \\
\hline$>100,00$ copies/ml, n. $(\%)$ & $79(42.9 \%)$ \\
\hline
\end{tabular}

Haemocromoctometric and flow cytometric analyses have been obtained in both groups of patients during the presentation of the respective disease, prior to the initiation of any treatments.

A total of 110 COVID-19 patients were included in the study. These patients referred to our Hospital during the actual SARS-CoV-2 outbreak from March to May 2020. They were 43 females and 67 males, with a $F / M=0.6$. They were all adults, with a medium age of 69 years $\pm 12.2(\min .35$, max 94$)$. The diagnosis was based on the positive result at rRT-PCR test, performed on the nasopharyngeal and oropharyngeal specimens.

A total of 184 HIV patients were included in the study. These patients referred to our University Hospital during the last 23 years, from 1999 to 2019 . They were 28 females and 156 males ( $F / M=0.2)$. They were all adults, with a medium age of 40 years \pm 11.4 ( $\min .21$, max 69$)$. The diagnosis was based on the result of HIV viral load test at admission. The value of HIV viral load was very high in all of them, with a medium value of 377,696 copies $/ \mathrm{ml}$. In 105 cases (57.1\%) the viral load was $<100,000 \mathrm{copies} / \mathrm{ml}$. In 79 of them $(42,9 \%)$ the viral load was $>100,000$ copies $/ \mathrm{ml}$. There is a correlation between the HIV viral load and the level of CD4+ T-cells (Fig. 1).

Such correlation is more evident in patients with a HIV viral load $<100,000$ copies/ml. No correlation is observed between HIV viral load and CD8+ T cells count (data not shown). Blood was drawn from all of 
them at the time of diagnosis for haemocrocytometric and flow cytometric analyses. Data have been retrospectively retrieved, collected and compared to those obtained from COVID-19 patients.

\section{Hemocromocytometric data}

When we compare the mean values of the haemocrocytometric parameters in AIDS and COVID-19 patients we find that there are some relevant differences (Table 2) (Fig. 2).

Table 2. Haemocromocytometric and Flow Cytometric indices in COVID-19 and AIDS patients 


\begin{tabular}{|c|c|c|c|c|}
\hline & $\begin{array}{l}\text { Normal } \\
\text { values }\end{array}$ & $\begin{array}{l}\text { COVID-19 n. = } \\
110\end{array}$ & AIDS n. $=184$ & $\begin{array}{l}P \\
\text { value }\end{array}$ \\
\hline \multicolumn{5}{|l|}{ Haemocromocytometric indices } \\
\hline RBC (106/ml) & $3.9-5.2$ & $4.2 \pm 1.1$ & $4.7 \pm 0.6$ & $\begin{array}{l}<< \\
0,005\end{array}$ \\
\hline $\mathrm{HGB}(\mathrm{g} / \mathrm{dl})$ & $12.0-16.0$ & $12.2 \pm 1.8$ & $13.8 \pm 2.7$ & $\begin{array}{l}<< \\
0,005\end{array}$ \\
\hline HCT (\%) & $42-52$ & $36.6 \pm 5.6$ & $40.7 \pm 6.2$ & $\begin{array}{l}<< \\
0,005\end{array}$ \\
\hline WBC (103/ml) & $4.3-10.8$ & $8.4 \pm 4.6$ & $5.6 \pm 2.2$ & $\begin{array}{l}<< \\
0,005\end{array}$ \\
\hline Neutrophils (103/mm3) & $1.5-7.5$ & $6.7 \pm 4.3$ & $3.3 \pm 1.8$ & $\begin{array}{l}<< \\
0,005\end{array}$ \\
\hline Neutrophils (\%) & $45-74$ & $76.3 \pm 15.0$ & $57.8 \pm 12.2$ & $\begin{array}{l}<< \\
0,005\end{array}$ \\
\hline Lymphocytes (103/mm3) & $1.0-4.5$ & $1.1 \pm 1.3$ & $1.7 \pm 0.8$ & $\begin{array}{l}<< \\
0,005\end{array}$ \\
\hline Lymphocytes (\%) & $16-45$ & $15.2 \pm 10.5$ & $31.0 \pm 10.5$ & $\begin{array}{l}<< \\
0,005\end{array}$ \\
\hline N/L Ratio & $0.78-3.53$ & $10.2 \pm 17.2$ & $2.5 \pm 2.8$ & $\begin{array}{l}<< \\
0,005\end{array}$ \\
\hline Monocytes (103/mm3) & $0.2-1.0$ & $0.5 \pm 0.3$ & $0.4 \pm 0.3$ & 0.02 \\
\hline Monocytes (\%) & $2.0-10.0$ & $6.6 \pm 3.4$ & $7.9 \pm 4.1$ & 0.03 \\
\hline Eosinophils (103/mm3) & $0.00-0.70$ & $0.06 \pm 0.1$ & $0.2 \pm 0.2$ & $\begin{array}{l}<< \\
0,005\end{array}$ \\
\hline Eosinophils (\%) & $0.0-5.0$ & $0.8 \pm 1.0$ & $3.0 \pm 3.3$ & $\begin{array}{l}<< \\
0,005\end{array}$ \\
\hline Basophils (103/mm3) & $0.00-0.20$ & $0.02 \pm 0.1$ & $0.02 \pm 0.02$ & N.S. \\
\hline Basophils (\%) & $0.0-2.0$ & $0.3 \pm 0.6$ & $0.4 \pm 0.3$ & N.S. \\
\hline PLT (103/ml) & $140-400$ & $210.2 \pm 93.9$ & $213.3 \pm 73.9$ & N.S. \\
\hline P/L Ratio & $75-199$ & $\begin{array}{l}275.56 \pm \\
203.32\end{array}$ & $\begin{array}{l}135.91 \pm \\
131.49\end{array}$ & $\begin{array}{l}<< \\
0,005\end{array}$ \\
\hline \multicolumn{5}{|l|}{ Flow Cytometric indices } \\
\hline Lymphocytes - CD45+ (cell/ml) & $1178-3262$ & $925.6 \pm 867.6$ & $\begin{array}{l}1635.4 \pm \\
836.4\end{array}$ & $\begin{array}{l}<< \\
0,005\end{array}$ \\
\hline
\end{tabular}




\begin{tabular}{|c|c|c|c|c|}
\hline $\begin{array}{l}\text { B Lymphocytes - CD45+/CD19+ } \\
\text { (cell/ml) }\end{array}$ & $200-400$ & $173.6 \pm 666.6$ & $155.0 \pm 125.9$ & N.S. \\
\hline T Lymphocytes - CD45+/CD3+ (cell/ml) & $1100-1700$ & $623.7 \pm 433.1$ & $\begin{array}{l}1294.5 \pm \\
721.0\end{array}$ & $\stackrel{<}{0,005}$ \\
\hline $\begin{array}{l}\text { T Lymphocytes - CD45+/CD3+/CD8+ } \\
\text { (cell/ml) }\end{array}$ & $500-900$ & $201.3 \pm 150.0$ & $903.2 \pm 555.9$ & $\stackrel{<}{<, 005}$ \\
\hline $\begin{array}{l}\text { T Lymphocytes - CD45+/CD3+/CD4+ } \\
\text { (cell/ml) }\end{array}$ & $700-1100$ & $396.9 \pm 317.0$ & $367.4 \pm 303.0$ & N.S. \\
\hline $\begin{array}{l}\text { T Lymphocytes - CD 45+/CD4+/CD8+ } \\
\text { Ratio }\end{array}$ & $1.0-1.9$ & $2.5 \pm 1.6$ & $0.5 \pm 1.3$ & $\begin{array}{l}<< \\
0,005\end{array}$ \\
\hline $\begin{array}{l}\mathrm{NK}-\mathrm{CD} 3+/ \mathrm{CD} 16+/ \mathrm{CD} 56+/ \mathrm{CD} 45+ \\
\text { (cell/ml) }\end{array}$ & $200-400$ & $108.4 \pm 83.3$ & $160.8 \pm 139.4$ & 0.0001 \\
\hline
\end{tabular}

Haemocromocytometric and flow cytometric indices in COVID-19 and AIDS patients. For each parameter the mean values \pm the standard deviations are reported. The $p$ values are indicated as a measure of the levels of significance of the differences among the two groups. NS = not significant.

In particular, COVID-19 patients show a moderate anemia, compared to AIDS patients, with lower levels of RBC, HGB and HCT. On the contrary, the absolute number of WBC is lower in patients with AIDS compared to those with COVID-19. The reduction in WBC, observed in AIDS patients, is mostly due to a moderate reduction in their absolute count of Neutrophils. Conversely, the mean absolute number of lymphocytes is higher in AIDS patients compared to those observed in COVID- 19 patients. As a consequence, the NLR is much higher in COVID-19 than in AIDS patients. We observe also a reduction of eosinophils in COVID-19 patients compared to AIDS ones, albeit at a lower level of statistically significance. No significant differences are present in the absolute numbers, of basophils, and platelets. Due to the more severe reduction in the absolute count of lymphocytes, observed in COVID-19 patients, as compared to AIDS patients, the PRL is higher in the former group with respect to the latter one.

\section{Flow Cytometry data}

The comparative study of the lymphocytes and their subpopulations by FCA in our AIDS and in COVID19 patients indicate that there are interesting similarities and relevant differences between the two groups of patients (Table 2) (Fig. 3).

SARS-CoV-2 virus infection is associated with a marked reduction in the CD45+ Lymphocyte cell populations, while HIV-infected patients show normal levels of CD45+ lymphocytes. Mean values of T cells expressing the CD $45+$ antigen in COVID-19 patients are, in fact, $56 \%$ of the corresponding values in AIDS patients (Table 2). The total count of B cells is slightly reduced in both groups of patients, but we don't observe any significant difference between them. A marked reduction in the CD4+ cells is considered the most distinctive laboratory characteristic of AIDS. Interestingly, we observe a similar 
reduction of the absolute count of this T cell subpopulation in COVID-19 patients too and we cannot detect statistically significant differences between the levels of this CD4+ lymphocytes subpopulation in the two groups of patients. Therefore, both SARS-CoV-2 and HIV appear to be able to induce the same effect on these cells. One of the most striking difference between the two groups of patients relies in the absolute number of CD8+ cells. As previously reported in the literature, the CD8+ cells are normal or slightly elevated in the serum of AIDS patients. On the contrary, in our COVID-19 patients, we observe a marked reduction in the levels of this T cells subpopulation. As a consequence of that, the CD4+/CD8+ ratio is much higher in COVID-19 patients, compared to AIDS patients. Representative images of FCA in SARS-CoV-2-infected and HIV-infected patients are reported in figure 4 (Fig. 4).

The absolute number of NK cells is reduced in both diseases, but this effect is more pronounced in COVID-19 patients compared to AIDS ones. Our results indicate that HIV and SARS-CoV-2 viruses are both capable of inducing a comparable reduction in the CD4+ lymphocyte subpopulation, but SARS$\mathrm{CoV}-2$ is much strongly associated with a marked reduction in the CD8+ lymphocyte subpopulation, a finding that appears to be specific for this virus, since it is not observed in HIV infected patients.

\section{Discussion}

There are relevant similarities between HIV and SARS-CoV-2 viruses. They are both RNA virus, able to cross species barriers and to transmit to humans, causing zoonotic diseases. Pandemic diffusion of these viruses has impacted people all over the world, spreading fear and uncertainty in the general population [7]. In addition, it has been reported that SARS-CoV-2 can infect T lymphocytes, the same cells targeted by HIV [8] and individuals with severe SARS-CoV-2 infection may exhibit lymphopenia just like HIV infected patients [9].

Lymphocyte subset analysis using monoclonal antibodies and FCA is a powerful tool. The evaluation of the immunophenotypic profile of a patient (i.e., the number of different types of lymphocytes determined by flow cytometry) is currently used in immunodeficiencies, leukemias and lymphomas as well as in posttransplanted patients. FCA has proved to be essential in providing information on the T cell CD4+ subset reduction, which is considered the laboratorial hallmark of the evolution of HIV infection as well as an indicator, together with clinical evidence of HIV infection, for the commencement of antiretroviral therapy. FCA provides the health care professional with information about the relative and absolute population of immune subtypes in the blood and has become the gold standard in estimating CD4+ counts for both diagnosis and monitoring of the response to therapy in HIV-infected patients. CD4+ T cells, in fact, are central mediators of immune response in humans. They play a crucial role in coordinating cellular and humoral immune responses against infections. The gradual loss of CD4+ T cells represents the hallmark of HIV and the consequent progressive impairment of immunity constitutes also a relevant cause of death in these patients.

It has been previously reported that the main difference in lymphoid phenotypes observed in HIV- infected patients is a reduction by $40 \%$ in the CD $4+T$ helper population and a $45 \%$ increase in the CD $8+$ cytotoxic 
T-cell population [10]. However, despite decades of experimental research, the complete mechanism of CD4+ T depletion in HIV infection still remains to be explained [11].

Growing amounts of data have accumulated and are available regarding the immune response in patients affected by COVID-19. Dysregulation of immune response, especially in T lymphocytes, are frequently observed in SARS-CoV-2 infection and might be highly involved in the pathological process of COVID-19. The common feature consisted, in fact, in lymphopenia, which can be severe and progressive in some patients, mostly the more severely affected and those with fatal illnesses $[3,12]$. In another study in which the COVID-19 patients have been stratified according their disease severity and data have been crossed with the composition of immune cells, an inverse correlation between disease severity and percentage of lymphocytes has been reported [9]. The same inverse correlation was reported with CD8+ T cells too [13]. There is evidence of an exuberant inflammatory response, similar to cytokine release syndrome, which was defined as "cytokine storm syndrome" [14].

The analysis of the lymphocyte subsets by flow cytometry represents an essential help in the early screening, diagnosis and treatment of COVID-19. The total number of B cells, T cells and NK cells are significantly decreased in patients with COVID-19 and such reduction is more evident in the severe cases compared to the non-severe ones. In patients with COVID-19 both helper T cells (CD4+) and suppressor T cells (CD8+) have been reported to be below normal levels, and the decline of helper T cells was more pronounced in severe cases.

The major difference that we have observed in our two groups of patients consists in the different number of T cells, analyzed by FCA. The HIV patients showed a marked reduction in the CD4+ $T$ cells and a consequent reduction in the CD4+/CD 8+ ratio. Surprisingly, a comparable reduction in the CD4+ T cell population is observed also in COVID-19 patients, with no statistically significant difference between the two diseases. In COVID-19 patients, the CD4+ reduction is associated with a marked reduction in the CD8 $+T$ cell population. As a consequence, the CD4+/CD8+ ratio is increased compared to normal values and much higher when compared to HIV patients.

The most evident difference between COVID-19 and AIDS is related to the absolute count of CD8+ T cells. The total number of the CD8+ T cell subpopulations, in fact, is strikingly different in the two groups of patients. In our HIV patients the CD8+ T cells are slightly increased, with respect to the normal value. In this regard, a remarkable expansion of CD8+CD28-CD127loCD39+ Treg cells, that correlates with HIV viremia, has been previously reported [15]. The total number of these cells correlates also with the clinical course of the disease and, in particular, with signs of chronic immune cell activation and with immunodeficiency events. Conversely, in our COVID-19 patients, the level of CD8+ T cells is markedly reduced. We are not able to confirm these data in our patients because they have not been evaluated using antibodies directed against the Treg cell population or their subgroups, namely the naïve-resting cells (CD45RA+) and the memory-activated cells (CD45RA-). In both virus infections, the exact reason of the reduction in the lymphocyte numbers in the blood is not clearly defined yet and many hypotheses have been proposed $[11,16]$. In particular, it is not clear whether the reduction in the serum levels of $T$ cells 
is due to an imbalance between production of T- cells and increased apoptosis of CD4+ T cells by viral attack the so-called of "accelerated destruction" hypothesis. Another possibility is that the CD4+ depletion could be due to an accelerated turnover of the CD4+ T cells, with increased production of activated CD4+ $T$ cells characterized by a very short life span. These cells are lost rather quickly due to activation-induced cell death or apoptosis, the so- called "hyper immune activation hypothesis". Another hypothesis is that the gradual loss of peripheral CD4+ T cells, observed during HIV infection, could be due to a massive production of proinflammatory cytokines, the so-called "cytokine storm", that has deleterious effects on $\mathrm{CD} 4+\mathrm{T}$ cells, thus leading to their clonal deletion. In addition, the occurrence of a highly inflammatory form of programmed cell death, called pyroptosis has been involved. In this type of apoptosis, the dying cell releases all its cytoplasmic contents, including inflammatory cytokines that, in turn, trigger pyroptosis in other T-cells as part of a vicious cycle of abortive T cell depletion. Finally, CD4 depletion has been associated with a relative expansion of Treg cells, irrespective of the presence or absence of circulating virus [17].

The depletion of CD4+ T cells may also be due to an altered lymphocyte trafficking [18]. In the case of HIV, it has been suggested that the depletion of CD4 lymphocytes could be due to an increased CD4+ lymphocyte homing rates [19], but the mechanism is not fully elucidated yet.

A clue to help in resolving this question may derive from the use of whole-body positron emission tomography (PET). The 18F-FDG PET/CT scanning has been demonstrated to be of benefit in determining the location and severity of disease activity in many inflammatory disorders [20]. It has been used in AIDS patients in search of specific sites of immune activation. Upon HIV infection, resting lymphocytes are activated and switch to glycolysis, increasing their glucose uptake by around 20 -fold over 24 hours [21,22]. In 2003, Alexander M Scharko et al. reported their attempts to identify the specific immune-system activation in response to HIV infection [23]. A total of 15 patients were analyzed in different stages of the disease. According to this study HIV-1 is able to induce activation of distinct lymphoid tissues according to the stages of the disease. When the analysis was performed in the acute stage, PET imaging showed that activated lymphoid tissues was localized in the head and neck, with some splenic involvement. Mid stages of the disease are associated with a more generalized pattern of peripheral lymph-node activation. Finally, during the late stages of the disease an involvement of abdominal lymph nodes was more evident. This study demonstrated that whole- body FDG PET images of HIV-positive patients present a clear association between the pattern of lymphoid tissue activation and HIV progression. Unfortunately, no study has been published so far concerning the pattern of PET imaging in COVID-19 patients.

Another relevant observation is related to the reduced number of NK cells, observed in both COVID- 19 and AIDS patients. NK cells play a fundamental role in the immune response, bridging the innate and adaptive immune systems. In particular, they are considered the antiviral effectors of the innate immune system. NK cells, in fact, are capable to directly respond to viruses, to develop memory-like responses after initial pathogen encounter or vaccination, and to shape the adaptive immune response. The role on NK cells in HIV infection has been recently reviewed [24] and these cells are gaining consideration in 
COVID-19 too [25]. The absolute number of NK cells is reduced in both our AIDS and COVID-19 patients. However, we observe a more severe reduction of NK cells in COVID-19 patients compared to AIDS ones. The meaning of this difference and the exact role of NK in HIV as well as in SARS-CoV-2 infections needs to be elucidated yet.

\section{Conclusion}

In conclusion, our study indicates that HIV/AIDS and SARS-CoV-2/COVID-19 have some unquestionable similarities and some relevant differences in their ability to elicit immune response in their hosts. Comparison of their effects on serum levels of lymphocytes subpopulations indicate that both viruses produce a comparable reduction in CD4+ $T$ cells, but they differ in their effects on the CD8+ $T$ cell subpopulation and NK cells. SARS-CoV-2 virus is associated with a marked reduction of CD8+ T cells and a more pronounced reduction of NK cells, whereas in HIV infected patients CD8+ cells are slightly elevated or normal and their NK cells are reduced compared to normal levels but less than those observed in COVID-19 patients. The reduction of the serum levels of these T cells subpopulations could be due to their homing at specific sites, as suggested by the results of FDG- PET/TC in HIV patients but no data are so far available in COVID-19 patients. These results indicate that immune system reacts differently to the two infections. The clarification of the differences and similarities between the two viruses will be useful to understand their mechanism of action and to design specific preventive and therapeutic approaches.

\section{Declarations}

\section{Acknowledgments}

We are deeply grateful to the COVID-19 Sant'Andrea Group and in particular to Prof. Luciano De Biase, Prof, Paolo Martelletti, to Prof. Andrea Laghi and to Prof Monica Rocco for their support in the presentation of the study for the approval by our Institutional Review Board. The study was funded by Sapienza University of Rome. The funder of the study had no role in study design, data collection, data analysis, data interpretation, or writing of the report. The corresponding authors had full access to all the data in the study and had final responsibility for the decision to submit for publication.

\section{Author Disclosure Statement}

No commercial association that might create a conflict of interest in connection with submitted manuscripts and no competing financial interests to declare.

\section{Corresponding author}

Salvatore Sciacchitano

Department of Clinical and Molecular Medicine, 
Sapienza University, Viale Regina Elena n. 324, 00161 Rome, Italy salvatore.sciacchitano@uniroma1.it

\section{References}

1. Mildvan, D. et al. Opportunistic infections and immune deficiency in homosexual men. Intern. Med. 96, 700-4 (1982).

2. Wu, Z. \& McGoogan, J.M. Characteristics of and Important Lessons From the Coronavirus Disease 2019 (COVID-19) Outbreak in China Summary of a Report of 72314 Cases From the Chinese Center for Disease Control and Prevention. JAMA Published online February 24, (2020) https://doi.org/10.1001/jama.2020.2648.

3. Huang, C. et al. Clinical features of patients infected with 2019 novel coronavirus in Wuhan, China. Lancet 395, 497-506 (2020). Epub 2020 Jan 24, doi: 1016/S0140-6736(20)30183-5.

4. Mehta, P. et al. COVID-19: consider cytokine storm syndromes and immunosuppression. Lancet 395, P1033-1034 (2020), Epub 2020 March 16, doi: 10.1016/S0140-6736(20)30628-0.

5. WHO, Laboratory testing for coronavirus disease (COVID-19) in suspected human Interim guidance 19 March 2020, https://apps.who.int/iris/rest/bitstreams/1272454/retrieve, accessed on June 29, 2020.

6. World Health Consolidated guidelines on HIV prevention, diagnosis, treatment and care for key populations - 2016 update. WHO website. http://apps.who.int/iris/bitstream/10665/246200/1/9789241511124-eng.pdf?ua=1, accessed on June 29, 2020.

7. Relf, V. What's Old is New! Similarities Between SARS-CoV-2 and HIV. J Assoc Nurses AIDS Care 31, 263-265 (2020).

8. Wang, et al. SARS-CoV-2 infects T lymphocytes through its spike protein-mediated membrane fusion. Cell Mol. Immunol. 1-3 (2020), published online ahead of print, 2020 Apr 7, doi:10.1038/s41423-0200424-9.

9. Tan, L. et al. Lymphopenia predicts disease severity of COVID-19: a descriptive and predictive study. Target Ther. 5, 33 (2020), published online ahead of print 2020 Mar 27, doi: 10.1038/s41392-0200148-4.

10. Tsoukas, C.M. \& Bernard, N.F. Markers predicting progression of human immunodeficiency virusrelated disease. Microbiol. Rev. 7, 14-28 (1994).

11. Vidya Vijayan, K., Karthigeyan, K.P., Tripathi, S.P. \& Hanna, L.E. Pathophysiology of CD4+ T-Cell Depletion in Hiv-1 and Hiv-2 infections. Front. Immunol. 8, 580 (2017), published 2017 May 23, doi:10.3389/fimmu.2017.00580.

12. Qin, C. et al. Dysregulation of Immune Response in Patients With Coronavirus 2019 (COVID-19) in Wuhan, China. Clin. Infect. Dis. 2020, ciaa248, published online ahead of print, doi: $10.1093 / \mathrm{cid} / \mathrm{ciaa} 248$. 
13. Zheng, M. et al. Functional exhaustion of antiviral lymphocytes in COVID-19 patients. Mol. Immunol. 17, 533-535 (2020).

14. Mehta, et al. COVID-19: consider cytokine storm syndromes and immunosuppression. Lancet 395, P1033-1034 (2020), Epub 2020 Mar 16, doi: 10.1016/S0140-6736(20)30628-0.

15. Fenoglio, D. et al. CD8+CD28-CD127loCD39+ regulatory T-cell expansion: A new possible pathogenic mechanism for HIV infection? Allergy Clin. Immunol. 141, 2220-2233 (2017), epub 2017 Nov 2, doi: 10.1016/j.jaci.2017.08.021.

16. Hazenberg, M.D., Hamann, D., Schuitemaker, H. \& Miedema, F. T cell depletion in HIV-1 infection: how CD4+ T cells go out of stock. Immunol. 1, 285-9 (2000), doi:10.1038/79724.

17. Foxall, R.B. et al. Memory and naive-like regulatory CD4+ T cells expand during HIV-2 infection in direct association with CD4+ T-cell depletion irrespectively of viremia. AIDS 25, 1961- 70 (2011), doi: 1097/QAD.0b013e32834b3554.

18. Rosenberg, Y.J., Anderson, A.O. \& Pabst, R. HIV-induced decline in blood CD4/CD8 ratios: viral killing or altered lymphocyte trafficking? Today 19, 10-17 (1998).

19. Chen, J.-Y. et al. CD4 lymphocytes in the blood of HIV+ individuals migrate rapidly to lymph nodes and bone marrow: support for homing theory of CD4 cell depletion. J. Leukoc. Biol. 72, 271- 278 (2002).

20. Glaudemans, W. et al. The Use of 18F-FDG-PET/CT for Diagnosis and Treatment Monitoring of Inflammatory and Infectious Diseases. Clin. Develop. Immunol. 2013, 623036 (2013), epub 2013 Aug 21, doi: 10.1155/2013/623036.

21. Bental, M. \& Deutsch, C. Metabolic changes in activated T cells: an NMR study of human peripheral blood lymphocytes. Reson. Med. 29, 317-326 (1993).

22. Marjanovic, S., Skog, S., Heiden, T., Tribukait, B. \& Nelson. B.D. Expression of glycolytic isoenzymes in activated human peripheral lymphocytes: cell cycle analysis using flow cytometry. Cell Res. 193, 425431 (1991).

23. Scharko, A.M. et al. Whole-body positron emission tomography in patients with HIV-1 infection. Lancet 362, 959-961 (2003).

24. Scully, E. \& Alter, G. NK Cells in HIV Disease. HIV/AIDS Rep. 13, 85-94 (2016).

25. Antonioli, L., Fornai, M., Pellegrini, C. \& Blandizzi, C. NKG2A and COVID-19: another brick in the wall. Mol. Immunol. 17, 672-674 (2020).

\section{Figures}


CD4+

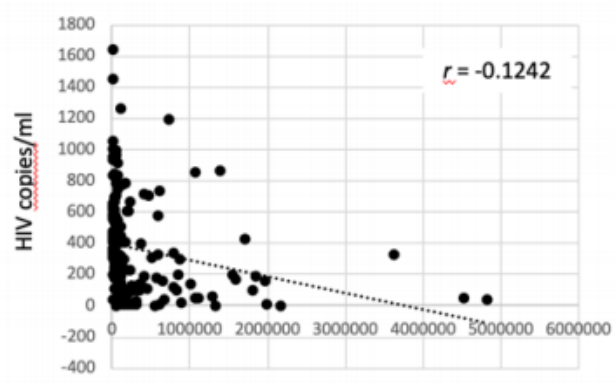

CD4+

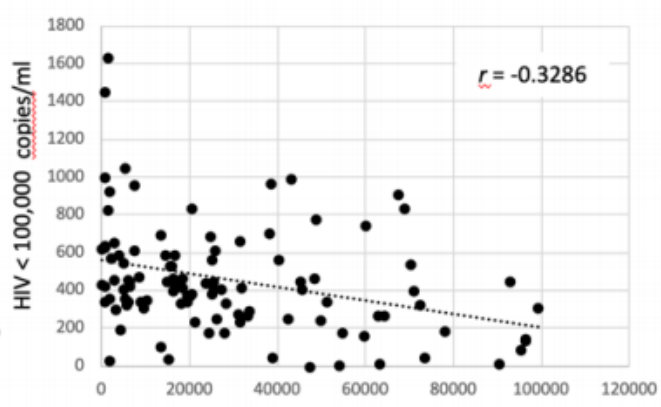

CD4+

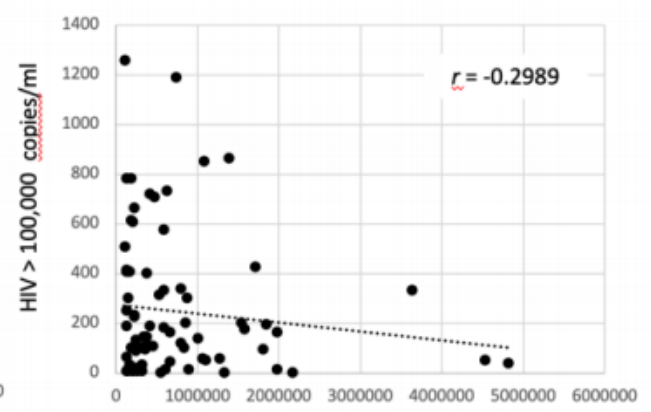

Figure 1

The HIV viral load and the absolute count of CD4+ T cells. Correlation between the absolute count of CD4+ T cells in serum and the HIV viral load in all patients, in those with $<100,000$ copies/ml and in those with $>100,000$ copies/ml. The coefficient of correlation $r$ is indicated.

Fig. 2
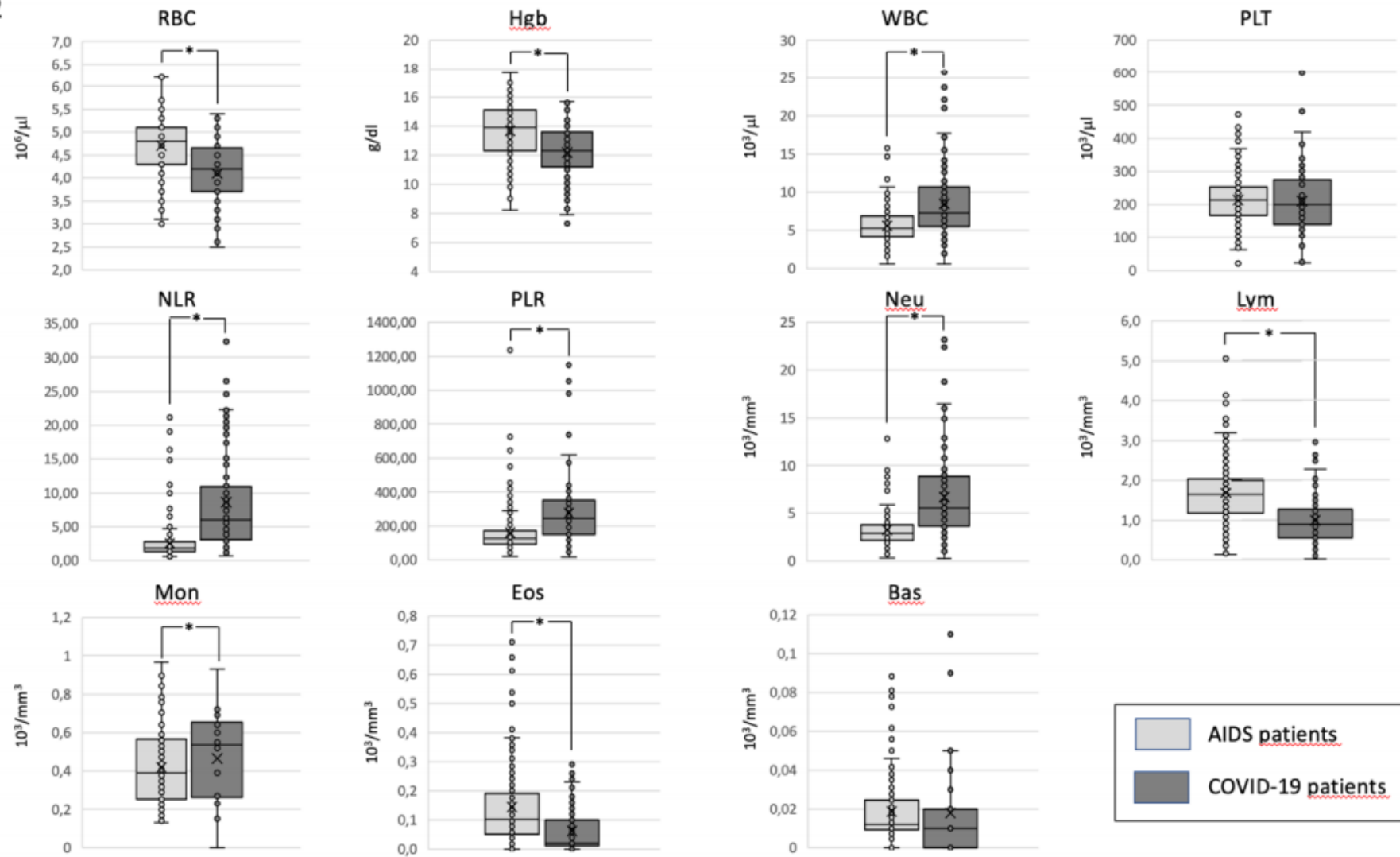

AIDS patients

COVID-19 patients

\section{Figure 2}

The haemocromocytometric indices. Box plots of haemocromocytometric indices in SARS-CoV-2-infected patients (dark gray), compared to HIV-infected patients (light gray). * indicates a statistically significance differences $(p<0.05)$. 
Fig. 3
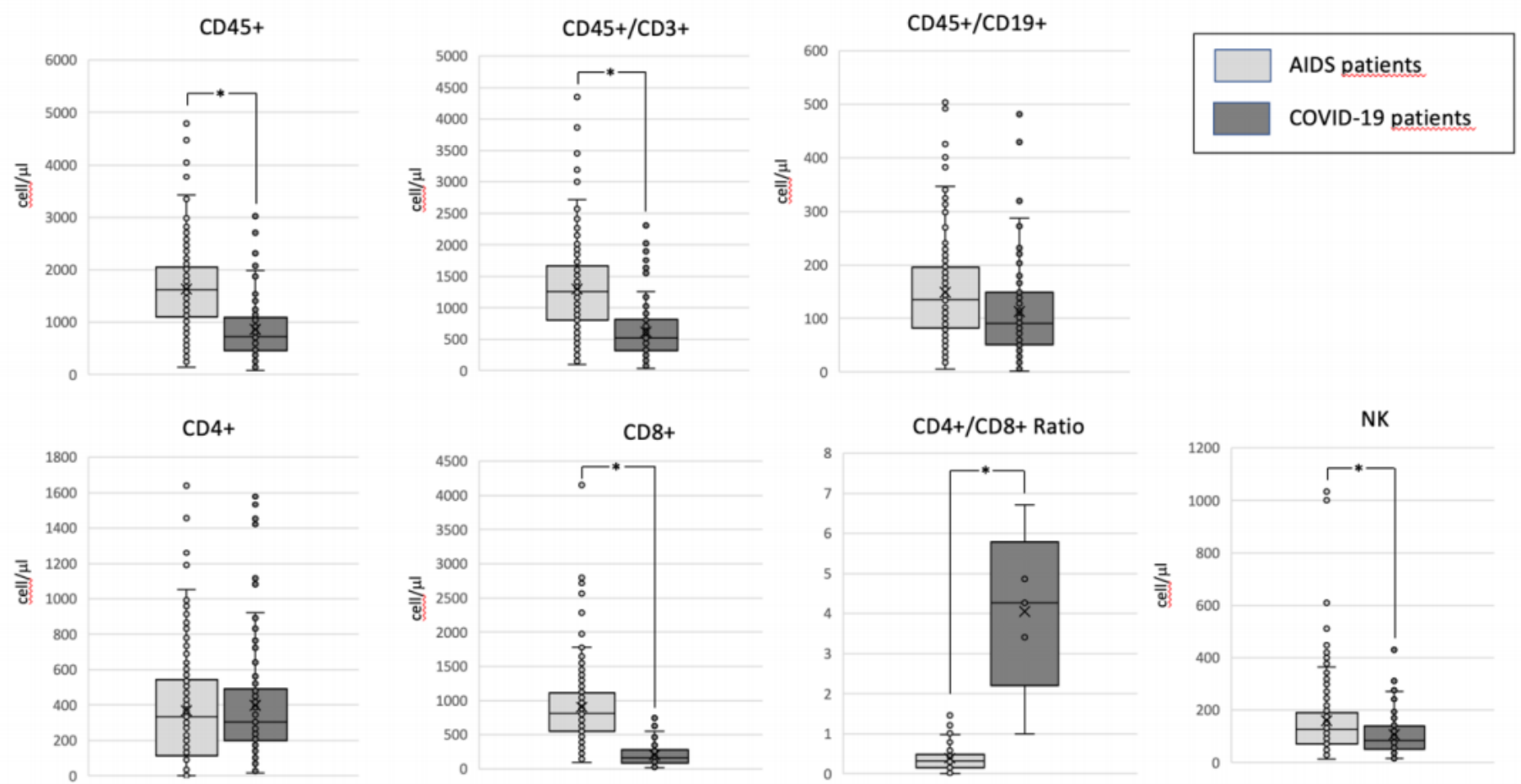

Figure 3

The flow cytometric indices. Box plots of flow cytometric indices in SARS-CoV-2-infected patients (dark gray), compared to HIV-infected patients (light gray). * indicates a statistically significance differences ( $p$ $<0.05)$.

Fig. 4
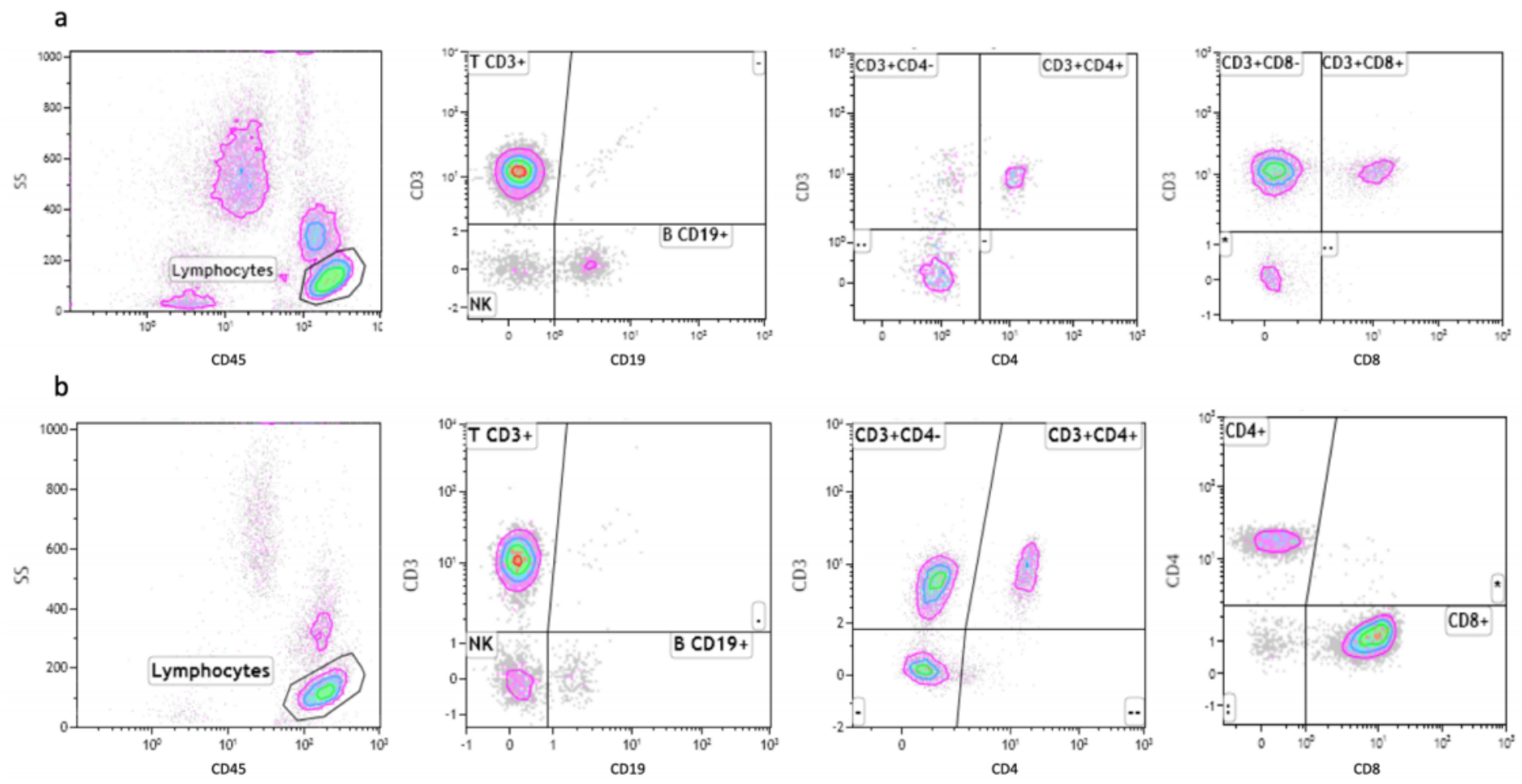


\section{Figure 4}

Representative images of Flow Cytometric Analysis (FCA) in SARS-CoV-2-infected and HIV-infected patients. a) FCA using fluorescently-tagged monoclonal antibodies specific for CD45+, CD19+, CD8+ and CD4+ in SARS-CoV-2-infected patients. b) FCA using fluorescently-tagged monoclonal antibodies specific for CD45+, CD19+, CD8+ and CD4+ in HIV-infected patients. 